ISSN 1997-342X (Online), ISSN 1991-8631 (Print)

Original Paper http://ajol.info/index.php/ijbes http://indexmedicus.afro.who.int

\title{
Estimation de l'impact en santé publique de la chimioprévention du paludisme saisonnier au Niger
}

\author{
Issa SALISSOU ${ }^{1,2}$, Lamine MAHAMAN MOUSTAPHA ${ }^{3}$, Ibrahim ALKASSOUM ${ }^{4}$, \\ Djakou HADIZA ${ }^{5}$ et Maman Laminou IBRAHIM ${ }^{3 *}$ \\ ${ }^{I}$ Institut de Santé Publique de Niamey, Niger; \\ ${ }^{2}$ Hôpital de District de Magaria, Niger; \\ ${ }^{3}$ Centre de Recherche Médicale et Sanitaire de Niamey, Niger; \\ ${ }^{4}$ Direction de la Surveillance et de la Riposte aux Epidémies, Niger; \\ ${ }^{5}$ Programme National de Lutte Contre le Paludisme. Niamey, Niger; \\ *Auteur correspondant ; E-mail : lamine@cermes.org ; 634 Bd de la Nation, \\ YN 034 Niamey Niger. BP : 10.887. Tel +227 20752040.
}

\section{RESUME}

La Chimioprévention du Paludisme Saisonnier (CPS) est une stratégie recommandée par l'Organisation Mondial de la Santé (OMS) dans les pays du Sahel. Une étude cas témoin a été conduite pour estimer l'impact en santé publique de la phase pilote de la CPS à Magaria au Niger en comparant l'incidence du paludisme de six Centres de Santé Intégrés (CSI) où la CPS est mise en œuvre à celle de 6 autres CSI sans CPS. Une enquête ménage a permis d'échantillonner 482 enfants dont 241 cas et 241 témoins. La CPS réduit le portage et la charge parasitaire de façon significative. La CPS réduit l'incidence du paludisme simple de $73 \%$. Il existe une corrélation forte entre la CPS et la réduction du nombre d'épisode de paludisme $(\mathrm{R}=0,59)$. La CPS réduit l'incidence du paludisme grave de $26,5 \%$. La CPS réduit les convulsions de $19,9 \%$ mais elle n'a pas d'impact sur l'anémie. La CPS réduit significativement le coma de $13,2 \%$ et les hospitalisations de $23 \%$. La CPS réduit enfin la mortalité de 48,3\%. La CPS est une stratégie prometteuse qui permettra aux pays du sahel de contrôler le paludisme et d'accéder au stade de pré-élimination en réduisant le portage, la morbidité et la mortalité.

(C) 2017 International Formulae Group. All rights reserved.

Mots clés : Impact, chimioprévention du paludisme saisonnier, Niger.

\section{Estimation of public health impact of seasonal malaria chemoprevention in Niger}

\section{ABSTRACT}

Seasonal Malaria Chemoprevention (SMC) is a new strategy recommended by the World Health Organization (WHO) to Sahel countries. A case-control study was conducted to estimate the public health impact of SMC pilot phase in Magaria, Niger by comparing the malaria incidence of six Integrated Health Centers (ICS) where SMC is implemented to 6 other CSIs without SMC. A household survey sampled 482 children, including 241 cases and 241 controls. Strong correlation between SMC and reduction of malaria episods was notifed $(\mathrm{R}=0.59)$ as well as the reduction of the incidence of uncumplicated maralaria $(37 \%)$ and 
severe malaria (26,5\%). Furthermore, SMC reduced convulsions by $19,9 \%$ but no impact on anemia. SMC significantly reduced coma by $13.2 \%$ and hospitalizations by $23 \%$. Finally, CPS reduced mortality by $48.3 \%$. SMC is a promising strategy that will enable the countries of Sahel to control malaria and reach the preelimination stage by reducing portage, morbidity and mortality.

(C) 2017 International Formulae Group. All rights reserved.

Keywords: Impact, Seasonal malaria chemoprevention, Niger.

\section{INTRODUCTION}

Depuis mars 2012, l'OMS a recommandé une nouvelle stratégie appelée Chimioprévention du Paludisme Saisonnier pour prévenir le pic de morbidité et de mortalité chez les enfants de moins de cinq ans des pays de la région du Sahel (OMS, 2013). Elle consiste à l'administration intermittente d'un traitement complet de Sulfadoxine Pyriméthamine + l'Amodiaquine pendant la saison de haute transmission. L'objectif est de maintenir des concentrations thérapeutiques de ces médicaments dans le sang pendant la période où le risque de contracter le paludisme est plus élevé (Carneiro et al., 2010). A l'instar des autres pays du sahel, le Niger a révisé sa politique nationale de lutte contre le paludisme en 2013 en introduisant la CPS. Cette stratégie a été mise en œuvre au niveau de sept districts sanitaires pilotes: Guidan Roumdji, Dakoro, Bouza, Madaroufa, Madaoua, Aguié et Magaria en collaboration avec des ONG comme Médecins Sans Frontière (MSF). C'est dans ce contexte qu'une évaluation de l'impact en santé publique de la CPS a été réalisée en comparant au niveau du district sanitaire de Magaria (Niger), l'incidence du paludisme de 6 CSI où la CPS est mise en œuvre à celle de 6 autres CSI où la CPS n'est pas mise en œuvre. L'objectif général étant d'estimer l'impact en santé publique de la Chimioprévention du Paludisme Saisonnier chez les enfants de 3 à 59 mois. Il s'agit de façon spécifique de déterminer le portage asymptomatique et la densité parasitaire moyenne chez les enfants de 3 à 59 mois, déterminer l'incidence du paludisme simple et la fréquence des épisodes, déterminer l'incidence des différentes formes du paludisme grave puis la fréquence des hospitalisations et enfin déterminer la mortalité attribuable au paludisme dans les deux sites.

\section{MATERIEL ET METHODES \\ Type d'étude}

Il s'agit d'une étude cas témoin afin d'évaluer l'apport en santé publique de la CPS, six mois après sa mise en œuvre à Magaria, au courant de la saison de haute transmission 2013. Cette étude a consisté en une enquête ménage ponctuelle et un suiviévaluation des informations sanitaires habituelles des CSI de Magaria.

\section{Site d'étude}

Cette étude est conduite au niveau du district sanitaire de Magaria, situé dans la partie Sud de la région de Zinder. Il couvre une superficie de $7903 \mathrm{Km}^{2}$. Le paludisme est endémique avec un pic en saison des pluies (juillet à septembre). Les six sites ayant fait l'objet de la CPS sont: Bangaza, Dantchiao, Dogo Dogo, Dungas, Maagaria et Mallaoua (Colorés en vert). Les six CSI sans CSP sont: Bandé, Baraguini, Kakitama, Takey, Wacha et Yekoua (Colorés en violet). (Figure 1). 


\section{Population d'étude}

La population d'étude est constituée par les enfants de 3 à 59 mois et les médecins chefs du district sanitaire de Magaria. En 2013, la population du district est estimée à 744268 habitants avec une densité de 94 habitants au $\mathrm{Km}^{2}$. En 2012, les formations sanitaires de ce district ont enregistré 124388 cas de paludisme dont 88189 cas chez les enfants de moins de 5 ans $(56,12 \%)$. Le taux de létalité dans les formations sanitaires est de $0,31 \%$.

Critères d'inclusion et non inclusion de l'étude

Sont inclus dans l'étude tous les enfants des deux sexes répondant aux critères suivants: être âgé de 3 à 59 mois, avoir bénéficié de la CPS, disposer de la carte bleue de CPS, résider dans les sites d'étude pendant la période de l'étude et enfin accepter le consentement éclairé.

\section{Critères de non inclusion}

Les critères de non inclusion de l'étude sont les suivants : être âgé plus de 59 mois, être hors de la zone d'étude, n'avoir pas reçu de CSP et enfin refuser le consentement éclairé.

\section{Echantillonnage}

Un échantillonnage en grappes à trois degrés a été utilisé. L'échantillonnage au $1^{\mathrm{er}}$ degré a été déterminé par tirage au sort sans remise. Sur la liste des villages du CSI, quatre (4) villages sont aléatoirement sélectionnés. Dix (10) ménages sont tirés dans chaque village sélectionné au $1^{\mathrm{er}}$ degré. Dans le cas des maisons à plusieurs ménages, il a été tiré aléatoirement un ménage. Tous les enfants âgés de 3 à 59 mois du ménage sont concernés.

\section{Calcul de la taille des échantillons}

La taille de l'échantillon est de 384 enfants de 3 à 59 mois soient 192 par bras. Cette taille est calculée sur la base qu'aucune étude sur la CPS n'a été faite. Nous avons pris une prévalence de $50 \%$ et une précision de $5 \%$ pour calculer la taille de l'échantillon. La formule utilisée $\mathrm{N}=\mathrm{Z} \alpha^{2}(1-\mathrm{p})^{*} \mathrm{p} / \mathrm{d}^{2}$.

\section{Collecte des données et outils}

Une goutte épaisse et un frottis mince ont été confectionnés à tous les enfants pour évaluer le portage parasitaire. Les lames sont colorées au Giemsa et lues au microscope. La collecte des données a été faite en administrant des questionnaires aux mères des enfants de 3 à 59 mois et aux médecins chefs des CSI au niveau des deux sites. Les outils de collecte sont constitués par des fiches d'enquêtes et un bulletin d'examen de laboratoire.

\section{Analyse des données}

Toutes les données des fiches d'enquêtes ont été saisies dans un fichier Excel. Les données ont été analysées grâce au logiciel Epi Info version 3.2 de 2010. Les tests de $\chi^{2}$ et Fischer exact ont été utilisés pour comparer les fréquences avec un seuil de précision de $5 \%$. Une régression linéaire a permis d'apprécier le lien entre le nombre d'épisodes de paludisme et la chimioprévention du paludisme saisonnier.

\section{Les directives éthiques}

Le projet a été présenté au comité national consultatif d'éthique qui l'a approuvé. Un consentement éclairé a été obtenu des parents ou tuteurs légaux des enfants inclus dans l'étude. 


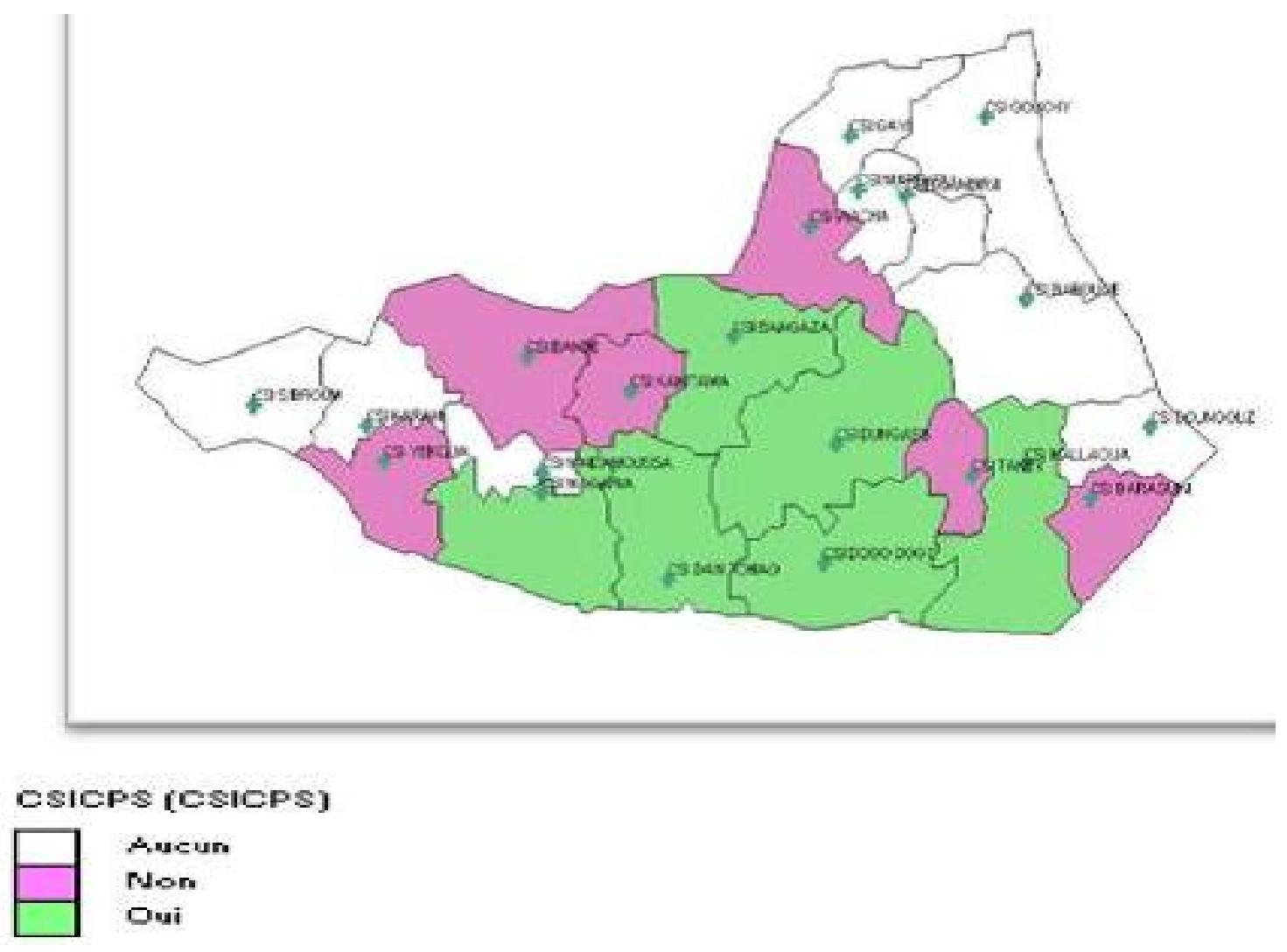

Figure 1 : Carte du district sanitaire de Magaria.

\section{RESULTATS}

\section{Caractéristiques d'échantillonnage}

Quatre cent quatre-vingt-deux (482) enfants, dont 241 au niveau des CSI où la CPS est mise en œuvre et 241 au niveau des CSI témoins ont été examinés. Les mères de ces 482 enfants ont été questionnées. Douze médecins chefs des CSI sont également questionnés dont 6 dans la zone avec CPS et 6 dans la zone témoin. L'âge moyen des enfants inclus dans l'étude était de 2,2 ans $(\alpha=1,32 ;[0,24 ; 5])$ et le sexe ratio de 1,06 en faveur des filles.

\section{Le portage asymptomatique, densité parasitaire moyenne et indice gamétocytaire}

Six mois après la mise en œuvre de la CPS à Magaria, seuls 23,2\% des enfants de moins de cinq ans étaient porteurs asymptomatiques de parasites dans la zone avec CPS contre $30,7 \%$ dans la zone témoin. La différence de portage asymptomatique était statistiquement significative entre les deux zones $(\mathrm{P}=0,03)$. La densité parasitaire moyenne était de $292 \mathrm{P} / \mu \mathrm{l} \quad(\alpha=114$, [200;600]) dans la zone avec CPS. Dans la zone témoin, la densité parasitaire moyenne était de $486 \mathrm{P} / \mu \mathrm{l}(\alpha=175,[200 ; 800])$. Il existe une différence statistiquement significative de 
charge parasitaire entre les deux zones $(\mathrm{P}<0,05)$. Aucun gamétocyte n'est trouvé dans les deux sites.

\section{Incidence du paludisme simple}

Seuls 58 cas de paludisme sont cliniquement dénombrés dans la zone avec CPS contre 234 cas dans la zone témoin. Seuls $24 \%$ des enfants de la zone avec CPS ont fait du paludisme contre $97 \%$ des enfants de la zone témoin. La CPS réduit l'incidence du paludisme simple de $73 \%$. Il existe une différence statistiquement significative de l'incidence du paludisme entre les deux zones $(\mathrm{P}<0,05)$.

\section{Distribution du nombre d'épisodes de} paludisme par enfant

Il y a eu 234 épisodes de paludisme dans la zone témoin contre seulement 58 épisodes de paludisme dans la zone à CPS. Dans la zone d'intervention les enfants ont fait en moyenne 0,4 épisode de paludisme contre 2,8 épisodes dans la zone témoin. Il existe une corrélation forte et positive entre la mise en œuvre de la CPS et la réduction du nombre d'épisode de paludisme chez les enfants de moins de cinq ans : $\mathrm{R}=0,59$.

\section{Incidence du paludisme grave}

Seuls 10,3\% des enfants de moins de cinq ans de la zone d'intervention ont manifesté des signes du paludisme grave. Par contre, $36,8 \%$ des enfants de la zone témoin ont fait du paludisme grave. Il existe une différence statistiquement significative de l'incidence du paludisme grave dans les deux zones $(\mathrm{P}=0,0002)$.

\section{Distribution des convulsions}

Seuls $10,3 \%$ des enfants de moins de cinq ans ont fait une crise convulsive dans la zone avec CPS. Par contre, 30,3\% ont fait des convulsions dans la zone sans CPS. Il existe une différence statistiquement significative de convulsion entre les deux zones (Fisher Exact $\mathrm{P}=0,002)$.

\section{Distribution des comas}

Trois virgule quarante-cinq pourcent $(3,45 \%)$ des enfants ont fait le coma dans les CSI avec CPS tandis que $16,6 \%$ ont fait le coma dans la zone sans la CPS. Il existe une différence statistiquement significative de coma entre les deux zones (Fisher Exact $\mathrm{P}=$ 0,009).

\section{Distribution de l'anémie}

Un virgule soixante-douze pourcent $(1,72 \%)$ d'enfants étaient anémiés dans les CSI avec CPS contre $1,71 \%$ dans la zone témoin. Il n'existe pas de différence statistiquement significative d'anémie entre les deux zones (Fisher Exact $\mathrm{P}=0,6$ ).

\section{Distribution des hospitalisations}

Seuls $5,1 \%$ des enfants ont été hospitalisés dans le groupe des enfants des villages ayant bénéficié de la CPS. Par contre, 28,2\% des enfants ont été hospitalisés dans les villages sans CPS. Il existe une différence statistiquement significative d'hospitalisation entre les deux zones (Fisher exact: $\mathrm{P}=$ 0,0002).

\section{Distribution des décès attribuables au paludisme}

Sept (7) enfants, soient 2,9\% sont décédés dans la zone avec CPS. Par contre, dans la zone témoin, il y a 13 enfants soient $5,3 \%$ de décès. Pour la prévalence des décès attribuables au paludisme, il y a deux (2) décès sur les 7 dans les CSI avec CPS, ce qui représente $28,57 \%$ des décès totaux. Dans la zone témoin, 10 décès sur 13 sont dus au paludisme. Ce qui représente $76,92 \%$ des décès totaux. La CPS réduit la mortalité attribuable au paludisme de 48,3\%. L'ensemble des résultats de l'étude sont résumés dans le Tableau 1. 
Tableau 1 : Tableau récapitulatif de l'impact de la CPS.

\begin{tabular}{lcccc}
\hline Caractéristiques & CSI avec PCS & CSI Témoins & ODDS RATIO & P-value \\
\hline Portage Parasitaire & $23,20 \%$ & $30,70 \%$ & $7,5 \%$ & $\mathrm{P}=0,03$ \\
Densité Parasitaire moyenne & $292 \mathrm{P} / \mu \mathrm{l}$ & $486 \mathrm{P} / \mu \mathrm{l}$ & - & $\mathrm{P}=0,000$ \\
Indice Gamétocytaire & 0 & 0 & - & $\mathrm{P}=1$ \\
Incidence du paludisme simple & $24 \%$ & $97 \%$ & $73 \%$ & $\mathrm{P}=0,000$ \\
Nombre d'épisode de paludisme & 0,4 & 2,8 & 2,4 & $\mathrm{P}=0,000$ \\
Incidence du paludisme grave & $10,30 \%$ & $36,80 \%$ & $26,5 \%$ & $\mathrm{P}=0,0002$ \\
Distribution des convulsions & $10,30 \%$ & $30,30 \%$ & $20 \%$ & $\mathrm{P}=0,002$ \\
Distribution du coma & $3,45 \%$ & $16.6 \%$ & $13 \%$ & $\mathrm{P}=0,009$ \\
Distribution de l'anémie & $1,72 \%$ & $1.71 \%$ & $0,01 \%$ & $\mathrm{P}=0,6$ \\
Hospitalisation & $5,10 \%$ & $28,20 \%$ & $23 \%$ & $\mathrm{P}=0,0002$ \\
Distribution des décès paludisme & $28,57 \%$ & $76,92 \%$ & $48 \%$ & $\mathrm{P}=0,052$ \\
\hline
\end{tabular}

\section{DISCUSSION}

Cette étude cas témoin évalue l'impact en santé publique de la CPS au Niger. C'est aussi la première étude d'impact sur la morbidité et la mortalité attribuable au paludisme après la mise en œuvre de la CPS au niveau du site pilote de Magaria. Les résultats de l'étude montrent que la CPS réduit significativement le portage, la morbidité et la mortalité dans les zones où elle est mise en œuvre. La CPS réduit non seulement le portage parasitaire mais aussi et surtout la charge parasitaire de manière significative. En effet, cette stratégie consiste à l'administration d'une dose curative de deux antipaludiques de demi-vie différente: L'amodiaquine dont la demi-vie est de sept jours et l'association sulfadoxine-pyriméthamine dont la demi-vie plasmatique est d'environ un mois. Cela permet de contrôler le portage parasitaire et la biomasse parasitaire en dessous du seuil pyrogénique. Aucun gamétocyte n'est aussi trouvé chez les enfants examinés. La CPS réduirait-elle la transmission du paludisme?
Une diminution des infections palustres de 73\% par la CPS fut observée au Burkina (Konaté et al., 2011).

L'une des caractéristiques notables de cette étude est de montrer que la CPS réduit l'incidence du paludisme de $73 \%$. En effet, en réduisant la densité parasitaire à un seuil inférieur au seuil pyrogénique, la CPS a un impact direct sur la morbidité du paludisme non compliqué. Ce résultat est proche de celui des études de l'OMS réalisées de 2002 à 2011 qui ont montré que la CPS permet d'éviter environ $75 \%$ de l'ensemble des accès palustres. Il s'apparente aussi à ceux de Dicko et al. (2008) au Mali qui ont trouvé une réduction de $67,5 \%$ entre la zone d'intervention et la zone témoin. Au Burkina, Konaté et al. (2011) ont trouvé que la CPS a réduit l'incidence du paludisme de $70 \%$.

La CPS réduit aussi significativement le nombre d'épisode de paludisme par enfant au courant de la saison de transmission. Il existe une corrélation forte et positive entre la CPS et la réduction du nombre d'épisode de 
paludisme: $\mathrm{R}=0,59$. Ces résultats sont comparables à ceux trouvés au Mali par Dicko et al. (2010) où ils ont trouvé 352 épisodes d'accès palustres dans les deux groupes dont 130 dans le groupe d'intervention et 221 dans le groupe contrôle. $\mathrm{Au}$ Sénégal, une étude conduite par l'IRD a montré que sur 1203 enfants âgés de moins de 5 ans, divisés en deux groupes de taille égale : un groupe traité et un témoin recevant un placebo, 39 épisodes de paludisme dans le groupe traité et 222 épisodes dans le groupe témoin, soit une efficacité protectrice de $86 \%$ (IRD, 2006). En Afrique tropicale, les enfants font plusieurs épisodes de paludisme par saison de pluie à cause de la forte transmission. La CPS permet de contrôler les infestations successives des moustiques chez les enfants de moins de cinq ans avant que ne s'installe la prémunition.

L'étude montre aussi que la CPS réduit l'incidence du paludisme grave de $26,5 \%$. Le paludisme simple évolue vers une forme grave s'il n'est pas rapidement pris en charge. Exceptionnellement, on peut observer subitement des formes graves. La réduction de l'incidence du paludisme simple entraîne la baisse de l'incidence du paludisme grave. Au Mali, Dicko et al. (2011) ont observé une efficacité protectrice de la CPS contre le paludisme grave de $87 \%$. Quant à Konaté et al. (2011) au Burkina, ils ont observé une réduction de $69 \%$ des cas graves de paludisme.

La CPS réduit significativement les convulsions de $19,9 \%$ et le coma de $13,2 \%$ chez les enfants de moins de cinq ans. Indépendamment de la forme clinique, beaucoup d'études ont montré que la CPS a un impact bénéfique sur l'incidence du paludisme grave. Cependant, rares sont les études qui rapportent sur l'impact de la CPS sur les convulsions et le coma.

Cette étude montre que la CPS n'a pas d'impact sur l'anémie. La recommandation de la CPS par l'OMS se fonde sur les résultats de sept études réalisées dans des zones de forte transmission saisonnière du paludisme dans les régions sahéliennes et Sub-sahariennes de l'Afrique subsaharienne entre 2002 et 2011. Ces études montrent que la CPS réduit probablement l'incidence de l'anémie modérée (OMS, 2013). Une enquête réalisée par MSF au Mali en 2012 a montré que les transfusions en pédiatrie ont connu une baisse de $25 \%$ après la mise en œuvre de la CPS. Chandramohan et al. (2005) montrent aussi que la CPS réduit l'anémie de $35,1 \%$ chez les enfants de moins de 15 mois mais n'a pas d'effet chez les enfants de 16 à 24 mois. Au Ghana, Cairns Matthew et al. (2008) ont montré que la CPS a une efficacité protectrice de 93,8\% contre l'anémie. Toutefois, cette protection est de courte durée. Par contre, Sesay et al. (2011) observent que la concentration d'hémoglobine ne varie pas et est respectivement de $10,2 \mathrm{~g} / \mathrm{dl}$ et $10,3 \mathrm{~g} / \mathrm{dl}$ dans le groupe d'intervention et dans le groupe contrôle. Ces résultats divergents montrent qu'en Afrique, l'anémie n'est pas essentiellement liée au paludisme et que d'autres facteurs comme la malnutrition joueraient un important rôle.

La CPS réduit significativement les hospitalisations dues au paludisme de $23 \%$. Cette proportion est inférieure à celle trouvée dans une enquête à domicile réalisée par MSF au Mali en 2012 où on note une baisse de $66,9 \%$ d'hospitalisations au niveau du centre de santé communautaire et de $69 \%$ au niveau du centre de référence. Au Ghana, le taux de réduction des admissions hospitalières après la 
mise en place de la CPS a baissé de 35,1\% (Chandramohan et al., 2005).

Cette étude montre que la CPS réduit la mortalité attribuable au paludisme de 48,3\%. Dans les études de l'OMS réalisées entre 2002 et 2011, la CPS a entraîné une diminution de la mortalité de l'enfant d'environ 1 pour 1000. Dicko et al. (2012) ont observé une baisse substantielle de la mortalité de $27 \%$ au Mali. Le taux de mortalité était de $2,8 \%$ dans la zone d'intervention contre $3,9 \%$ dans la zone témoin

\section{Conclusion}

Parmi les limites de l'étude, il faut citer la durée de l'étude qui est relativement courte pour une évaluation d'impact. En effet, les études d'impact doivent être relativement longues.

La CPS est une stratégie prometteuse qui permettra aux pays du sahel de contrôler le paludisme et d'accéder au stade de préélimination en réduisant significativement le portage, la morbidité et la mortalité attribuables au paludisme. Au vu d'importants résultats de la phase pilote, le programme national de lutte contre le paludisme au Niger peut mettre en œuvre à l'échelle nationale cette stratégie.

\section{CONFLIT D'INTERETS}

Les auteurs déclarent qu'il n'y a pas de conflit d'intérêt.

\section{CONTRIBUTIONS DES AUTEURS}

MLI a conçu le projet et rédigé l'article; LMM a participé la rédaction de l'article ; IS a participé à la mise en œuvre du projet; HD a aidé à trouver le financement ; IA a participé conception du projet et à la rédaction de l'article.

\section{REMERCIEMENTS}

Nous remercions le Médecin Sans Frontière Suisse pour avoir mis en œuvre la chimioprévention du paludisme dans le district de Magaria. Nous remercions également Yerima Bako, médecin chef de l'hôpital du District Sanitaire d'avoir permis la réalisation de cette étude dans son district. Nous remercions aussi l'administrateur de l'Institut de Santé Publique de Niamey qui a inscrit ce projet dans la liste de ses activités académiques comme sujets de mémoire du diplôme d'étude spécialisée en santé publique au titre de l'exercice 2013-2014.

\section{REFERENCES}

Bojang KA, Akor F, Conteh L, Webb E, Bittaye O, Conway DJ, Jasseh $\mathrm{M}$, Wiseman V, Milligan PJ, Greenwood B. 2011. Two Strategies for the Delivery of IPTc in an Area of Seasonal Malaria Transmission in The Gambia: A Randomised Controlled Trial. PLoS Med., $\quad$ 8: e1000409. doi:10.1371/journal.pmed.1000409

Cairns M, Carneiro I, Milligan P, OwusuAgyei S, Awine T, Gosling R, Greenwood B, Chandramohan D. 2008. Duration of Protection against Malaria and Anaemia Provided by Intermittent Preventive Treatment in Infants in Navrongo, Ghana. PLoS ONE, 3: e2227. doi:10.1371/journal.pone.0002227

Carneiro I, Smith L, Ross A, Roca-Feltrer A, Greenwood B, Schellenberg JA, Smith T, Schellenberg D. 2010. Intermittent preventive treatment for malaria in infants: a decision-support tool for subSaharan Africa. Bull. World Health Organ., 88: 807-814. doi:10.2471/BLT.09.072397

Chandramohan D. 2005. Cluster randomised trial of intermittent preventive treatment for malaria in infants in area of high, 
seasonal transmission in Ghana. BMJ 331: 727-733.

doi:10.1136/bmj.331.7519.727

Cissé B, Sokhna C, Boulanger D, Milet J, Bâ EH, Richardson K, Hallett R, Sutherland C, Simondon K, Simondon F, Alexander $\mathrm{N}$, Gaye O, Targett G, Lines J, Greenwood B, Trape J-F. 2006. Seasonal intermittent preventive treatment with artesunate and sulfadoxinepyrimethamine for prevention of malaria in Senegalese children: a randomised, placebo-controlled, double-blind trial. The Lancet, 367: 659-667. doi:10.1016/S0140-6736(06)68264-0

Dicko A, Diallo AI, Tembine I, Dicko Y, Dara N, Sidibe Y, Santara G, Diawara $\mathrm{H}$, Conaré T, Djimde A, Chandramohan, D, Cousens S, Milligan PJ, Diallo DA, Doumbo OK, Greenwood B. 2011 b. Intermittent Preventive Treatment of Malaria Provides Substantial Protection against Malaria in Children Already Protected by an Insecticide-Treated Bednet in Mali: A Randomised, DoubleBlind, Placebo-Controlled Trial. PLoS Med., $\quad$ 8: $\quad$ e1000407. doi:10.1371/journal.pmed.1000407

Dicko A, Konare M, Traore D, Testa J, Salamon R, Doumbo O, Rogier C. 2012. The implementation of malaria intermittent preventive treatment with sulphadoxine-pyrimethamine in infants reduced all-cause mortality in the district of Kolokani, Mali: results from a cluster randomized control trial. Malar. J., 11: 73. doi:10.1186/1475-2875-11-73

Dicko A, Sagara I, Sissoko MS, Guindo O, Diallo AI, Kone M, Toure OB, Sacko M, Doumbo OK. 2008. Impact of intermittent preventive treatment with sulphadoxine-pyrimethamine targeting the transmission season on the incidence of clinical malaria in children in Mali. Malar. J., 7: 123. doi:10.1186/14752875-7-123

IRD. 2006. Un nouvel espoir de traitement du paludisme pour les enfants - Institut de recherche pour le développement (IRD) [WWW Document], n.d. URL http://travail.ird.fr/la-mediatheque/ fiches-d-actualite-scientifique/235-unnouvel-espoir-de-traitement-dupaludisme-pour-les-enfants (accessed 4.11.17).

Konaté AT, Yaro JB, Ouédraogo AZ, Diarra A, Gansané A, Soulama I, Kangoyé DT, Kaboré Y, Ouédraogo E, Ouédraogo A, Tiono AB, Ouédraogo IN, Chandramohan D, Cousens S, Milligan, PJ, Sirima SB, Greenwood B, Diallo DA. 2011. Intermittent Preventive Treatment of Malaria Provides Substantial Protection against Malaria in Children Already Protected by an Insecticide-Treated Bednet in Burkina Faso: A Randomised, Double-Blind, Placebo-Controlled Trial. PLoS Med. 8: e1000408. doi:10.1371/journal.pmed. 1000408

OMS. 2013. Chimioprévention du paludisme saisonnier par administration de sulfadoxine-pyriméthamine et d'amodiaquine aux enfants: guide de terrain [WWW Document], n.d. . WHO. URL http://www.who.int/malaria/ publications/atoz/9789241504737/fr/ (accessed 4.11.17).

Sesay S, Milligan P, Touray E, Sowe M, Webb EL, Greenwood BM, Bojang KA. 2011. A trial of intermittent preventive treatment and home-based management of malaria in a rural area of The Gambia. Malar. J., 10: 2. doi:10.1186/1475-2875$10-2$ 